\title{
Development and validation of predictive model for lymph node metastasis in endometrial cancer: a SEER analysis
}

\author{
Xingchen $\mathrm{Li}^{1}$, Yuan Cheng ${ }^{1}$, Yangyang Dong ${ }^{1}$, Jingyi Zhou ${ }^{1,2}$, Zhiqi Wang ${ }^{1}$, Xiaoping Li $^{1}$, Jianliu Wang ${ }^{1,2}$ \\ ${ }^{1}$ Department of Obstetrics and Gynecology, Peking University People's Hospital, Beijing, China; ${ }^{2}$ Beijing Key Laboratory of Female Pelvic Floor \\ Disorders Diseases, Beijing, China \\ Contributions: (I) Conception and design: X Li, J Wang; (II) Administrative support: X Li; (III) Provision of study materials or patients: Y Cheng, Y \\ Dong; (IV) Collection and assembly of data: J Zhou, Z Wang; (V) Data analysis and interpretation: X Li; (VI) Manuscript writing: All authors; (VII) \\ Final approval of manuscript: All authors. \\ Correspondence to: Jianliu Wang. Department of Obstetrics and Gynecology, Peking University People’s Hospital, Beijing 100044, China. \\ Email: wangjianliu@pkuph.edu.cn.
}

\begin{abstract}
Background: The purpose of this study was to develop a nomogram that can be used to predict lymph node metastasis (LNM) in patients with endometrial cancer (EC).

Methods: The clinical data of EC patients diagnosed between 2004 and 2015 were retrieved from the Surveillance, Epidemiology, and End Results Program (SEER) registry. The nomogram was constructed using independent risk factors chosen by a multivariate logistic regression analysis. Accuracy was validated for both groups using discrimination analysis and calibration curves.

Results: The final study group consisted of 63,836 women that met specific inclusion criteria. The factors that were identified in the multivariate analysis to be significant predictors of LNM were age, tumor size, histological type, myometrial invasion, cervical stromal invasion, and tumor grade in training group $(\mathrm{N}=42,558)$. These variables were included in the nomogram. Discriminations of the nomogram and Mayo criteria were 0.848 (95\% CI: $0.843-0.853$ ) and 0.806 (95\% CI: 0.801-0.812), respectively. In the validation group ( $\mathrm{N}=21,278)$, the AUC values were 0.847 (95\% CI: $0.840-0.857)$ and 0.804 (95\% CI: 0.796-0.813) for the nomogram and the Mayo criteria, respectively $(\mathrm{P}<0.01)$. Calibration plots showed that training and validation cohorts were well-calibrated.

Conclusions: A nomogram was developed to predict LNM in EC patients based on a large populationbased analysis. The nomogram showed good performance for predicting LNM in patients with EC. This convenient predictive tool may help clinicians to formulate suitable individualized treatment.
\end{abstract}

Keywords: Endometrial cancer (EC); lymph node metastasis (LNM); nomogram; decision curve analysis

Submitted Jun 30, 2021. Accepted for publication Dec 10, 2021.

doi: $10.21037 /$ atm-20-5034

View this article at: http://dx.doi.org/10.21037/atm-20-5034

\section{Introduction}

Endometrial cancer (EC) is the most common gynecologic malignancy and the fourth most common of all tumor types in Western countries. According to the American Cancer Society, there are approximately 63,230 new cases and 11,350 related deaths of endometrial cancer each year in the United States (1). Symptoms such as abnormal vaginal bleeding usually present quickly after onset. Therefore, most EC patients are diagnosed at an early stage. Early diagnosis can allow for early treatment and good prognosis for EC patients. However, lymph node metastasis (LNM) is a major risk factor for recurrence and metastasis of EC. Para-aortic and pelvic LNM have been observed in 5\% and $9 \%$ of EC patients, respectively (2). According to the International Federation of Gynecology and Obstetrics (FIGO) staging system, this lymphatic involvement often indicates a poor prognosis (3). Disease-free survival (DFS) 
for patients at FIGO stages I and II was $90 \%$ for those without LNM, 75\% for those with pelvic LNM, and 38\% for those with para-aortic LNM (4). Another study reported a 5 -year overall survival rate of $53.6 \%$ for patients with para-aortal lymph nodal involvement, as opposed to $96.5 \%$ for those without any LNM (5).

A nomogram is a predictive tool that provides the overall probability of a specific outcome (6). Nomograms consist of multiple prognostic factors in a graphical display, to simply prediction of a specific event. In general, nomograms used for predicting patients' outcomes discriminate patients with a future event from those without. The performance of the nomogram is initially validated by providing discrimination and calibration values (7). Discrimination evaluates whether the model is able to discriminate patients (distinguish one patient from another) and is generally expressed with area under the ROC curve (AUC) values (8). Calibration describes how close predicted and actual outcomes are.

Many researchers have attempted to identify risk factors for LNM and predicted the probability of LNM by developing nomograms using different risk factors. A great number of clinicians followed the standard for judging low-risk groups without LNM proposed by the Mayo in 2000. The Mayo criteria categorizes EC patients as low-risk with the following characteristics: endometrioid endometrial cancer, tumor diameter $<2 \mathrm{~cm}$, grade 1 or 2 , and myometrial invasion (MI) $<50 \%$ (9). Mayo criteria has been estimated prospectively and widely used to guide the lymphadenectomy. For patients with low-risk, the 5 -year survival is almost $99 \%$ without lymph node dissection (10). Some studies also compared the Mayo criteria with other models, including nomograms designed as part of their studies $(11,12)$. However, according to the Mayo criteria, the rate of LNM for the high-risk group was $6.4 \%$, more than $70 \%$ patients without LNM were over-treated. What's more, due to the small size of patient populations, these studies could not provide crucial information on how small incremental changes in tumor size may affect patient outcomes. To date, there is no published model for predicting lymph node metastasis in EC patients based on a large cohort.

Because of this need, we designed a nomogram to predict LNM based on data from the Surveillance, Epidemiology, and End Results (SEER) database. In this study, we developed and internally validated a parametric model for predicting LNM. The model includes pathological characteristics based on a mathematical algorithm from a large population. Decision curve analysis was also used to estimate the clinical value of the nomogram and compare it with the Mayo criteria. We present the following article in accordance with the TRIPOD reporting checklist (available at http://dx.doi.org/10.21037/atm-20-5034).

\section{Methods}

\section{Patients and study design}

The National Cancer Institute's SEER database on cancer research is freely available to the public upon submission of a signed data-used agreement to the SEER administration. We extracted data for endometrial cancer cases diagnosed between 2004 and 2015 from the SEER registry to use for further analysis. Inclusion criteria for patients were as follows: age at diagnosis $>18$ years; and endometrial cancer was pathologically confirmed by histology (histological code: 8140-8389 for EEA, 8440-8499 for SEA). Patients who had a history of prior malignancy, with distant metastasis, or had missing information regarding lymph node metastasis, race, tumor size, histology, myometrial invasion, cervical stromal invasion, or tumor grade were excluded. A total of 63,836 patients in the SEER cohort meeting the criteria were selected for further analysis. Patients were then randomly divided into two groups in a 2:1 ratio, to form a training cohort $(\mathrm{N}=42,558)$ and an internal validation cohort $(\mathrm{N}=21,278)$. The flow chart used for data selection is shown in Figure 1. The ethics committee board of Peking University People's Hospital approved the use of patient data for this study. The study was conducted in accordance with the Declaration of Helsinki (as revised in 2013).

\section{Variables}

The patients' variables that were evaluated to design the nomogram were as following: age, race, tumor size, year of diagnosis, histological type, myometrial invasion, tumor grade, and lymph node metastasis. Age was categorized subjectively as $\leq 40,41-50,51-60,61-70$, or $>70$ years. Tumor size was classified as $\leq 2,2.1-5,5.1-10$, or $>10 \mathrm{~cm}$. The histological types were classified as endometrial endometrioid adenocarcinoma (EEA, ICD-O-3 codes: 8140-8389), serous endometrioid adenocarcinoma (SEA, ICD-O-3 codes: 8440-8499), or "other types". Patients were classified as low-risk using the Mayo criteria if they're symptoms were characterized by grade 1 or 2 tumors, less than $50 \%$ myometrial invasion, and tumor size $\leq 2 \mathrm{~cm}$. 


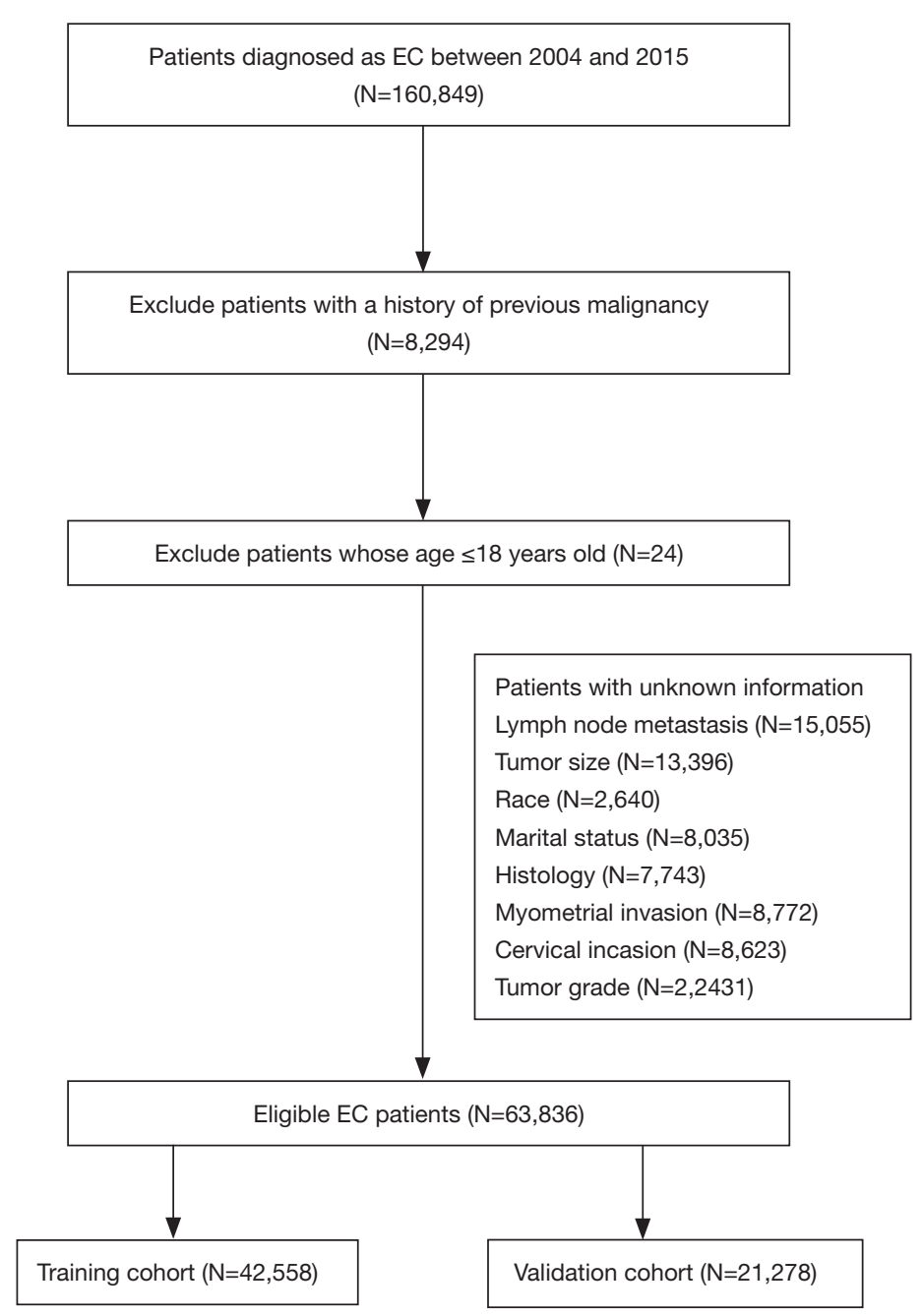

Figure 1 Flow chart for screening eligible patients in training and validation cohorts.

Patients classified as high-risk using the Mayo criteria had tumors with myometrial invasion $>50 \%$, grade 3 , and tumor size $>2 \mathrm{~cm}$.

\section{Statistical analyses}

All the categorical variables were described as frequencies and percentages. Univariate and multivariate logistic regression analyses were used to identify independent risk factors predictive of LNM and to develop the nomogram in the training cohort. All variables in the univariate analysis with $\mathrm{P}<0.05$ were considered statistically significant and selected for multivariate analysis. Candidate variables with $\mathrm{P}<0.05$ were selected using a backward stepwise selection from the full multivariate model. The nomogram was then constructed using these candidate variables.
The performance of the nomogram was assessed in both the training and validation groups by calculating discrimination and calibration criteria (13). Discrimination was quantified using an area under the receiver operating characteristic (ROC) curve. The area under the ROC curve (AUC) is a summary measure of the ROC that reflects the ability of a test to discriminate the outcomes across all possible levels of positivity. AUC values range from 0 to 1 , and a model is considered to have a poor, fair or good performance if the AUC value is between 0.5 to $0.6,0.6$ to 0.7 or greater than 0.7 , respectively. A calibration plot was generated to visualize how far the predictions were from the actual outcomes, displaying mean nomogrambased predictions in training and validation cohorts on the horizontal axis versus actually observed LNM probabilities.

Finally, decision curve analysis was performed to 
quantify the clinical usefulness of the model. Such analyses can determine the ability of a model to predict fine-scale outcomes based on a set of risk parameters. A model that performs well in the decision curve analysis has a higher net benefit than a model that simply classifies all patients as having the predicted outcome or no (zero) patients as having the outcome. Decision curve analysis can also be used to compare the net benefits of multiple models.

All analyses were performed by SPSS 21.0 and R software version 3.4.4 (https://www.r-project.org/), using the rms, presence/absence, and decision curve packages. $\mathrm{P}<0.05$ was considered statistically significant.

\section{Results}

\section{Clinical characteristics of patients}

The data from a total of 63,836 patients were included in the study. Out of the patients, 42,558 patients were placed within the training cohort, while 21,278 were placed within a validation cohort. Figure 1 showed a schematic of the screening process. The mean ages of patients within the training and validation sets were $62.41 \pm 11.62$ and $62.41 \pm 11.58$ years, respectively. Tumor size was $6.58 \pm 15.63 \mathrm{~cm}$ in the training cohort and $6.56 \pm 15.62 \mathrm{~cm}$ in the validation group. In the training cohort, most patients $(88.45 \%)$ were negative for LNM. The age of $3.49 \%$ patients were less than 40 years old, $10.11 \%$ patients were between 41 and 50 years old, $30.79 \%$ were between 51 and 60 years old, $31.86 \%$ were between 61 and 70 years old, and $23.75 \%$ were older than 71 years old. Most of the patients in both cohorts were white $(82.40 \%)$ and their tumor size was between 2 to $5 \mathrm{~cm}(49.36 \%)$. The pathological characteristics of the majority included EEA (87.27\%), no myometrial invasion (65.10\%), no cervical stromal invasion $(79.80 \%)$ and tumors classified as grade $1(41.50 \%)$. The two sets showed similar results for nearly all variables. Table 1 showed the details of demographic and pathological characteristics of the patients in the two cohorts.

\section{Risk factors for lymph node metastasis}

Our univariate analysis considered age at diagnosis, race, tumor size, histological type, myometrial invasion, cervical stromal invasion, and tumor grade as potential risk factors for LNM from the training cohort data. After multivariate logistic regression analysis, it was found that independent risk factors associated with LNM including age at diagnosis, tumor size, histological type, myometrial invasion, cervical stromal invasion, and tumor grade (Table 2). Among these independent risk factors, cervical stromal invasion was considered as a major predictor (OR $=6.09,95 \%$ CI: 5.50 6.76, $\mathrm{P}<0.001)$. Other factors considered to be predictors of LNM included age (OR $=1.01,95 \%$ CI: 1.01-1.02, $\mathrm{P}<0.001)$, tumor size $2-5 \mathrm{~cm}(\mathrm{OR}=1.51,95 \%$ CI: 1.34 $1.70, \mathrm{P}<0.001)$, tumor size $5-10 \mathrm{~cm}(\mathrm{OR}=2.71,95 \% \mathrm{CI}$ : 2.39-3.06, $\mathrm{P}<0.001)$, tumor size $\geq 10 \mathrm{~cm}(\mathrm{OR}=3.38,95 \%$ CI: 2.90-3.95, $\mathrm{P}<0.001)$, histological type SEA (OR =1.78, 95\% CI: $1.61-1.97, \mathrm{P}<0.001)$ and histological types other than EEA or SEA (OR $=1.33$, 95\% CI: $1.18-1.50, \mathrm{P}<0.001$ ), positive myometrial invasion ( $\mathrm{OR}=2.77,95 \% \mathrm{CI}: 2.18-3.52$, $\mathrm{P}<0.001$ ), tumor grade 2 ( $\mathrm{OR}=2.27,95 \% \mathrm{CI}: 2.04-2.53$, $\mathrm{P}<0.001)$ and tumor grade $3(\mathrm{OR}=4.68,95 \% \mathrm{CI}: 4.21-5.20$, $\mathrm{P}<0.001)$. Analysis in the validation set revealed the same independent risk factors for LNM (Table 3).

\section{Design and validation of the nomogram}

Based on the independent risk factors identified in the multivariate regression analysis, we designed a nomogram to predict LNM in EC patients (Figure 2). Among the variables considered in the predictive model, cervical stromal invasion was identified to be the most important predictive factor for the LNM nomogram. Point assignments and predictive scores for each variable in the nomogram models were calculated, with the total score corresponding to a predicted probability of LNM. The performance of the final model was assessed through discrimination and calibration analyses. Based on these analyses, the nomogram had an AUC value of 0.848 (95\% CI: $0.843-0.853)$ for the training group, as compared with 0.806 (95\% CI: 0.801-0.812) for the Mayo criteria ( $\mathrm{P}<0.01$; Figure $3 A$ ). In the validation group, the AUC value was 0.847 (95\% CI: $0.840-0.857$ ) for the nomogram and 0.804 (95\% CI: 0.796-0.813) for the Mayo criteria, respectively $(\mathrm{P}<0.01$; Figure $3 B)$. The nomogram showed discrimination majority to the Mayo criteria for both the training and validation cohorts. The calibration curves for predicting LNM demonstrated that the nomogram was well-calibrated for both the training (Figure $4 A$ ) and validation groups (Figure 4B).

\section{Optimal threshold of the nomogram}

Each patient was assigned a score using the calibrated 
Table 1 The demographics and pathological characteristics of patients in training and validation cohorts

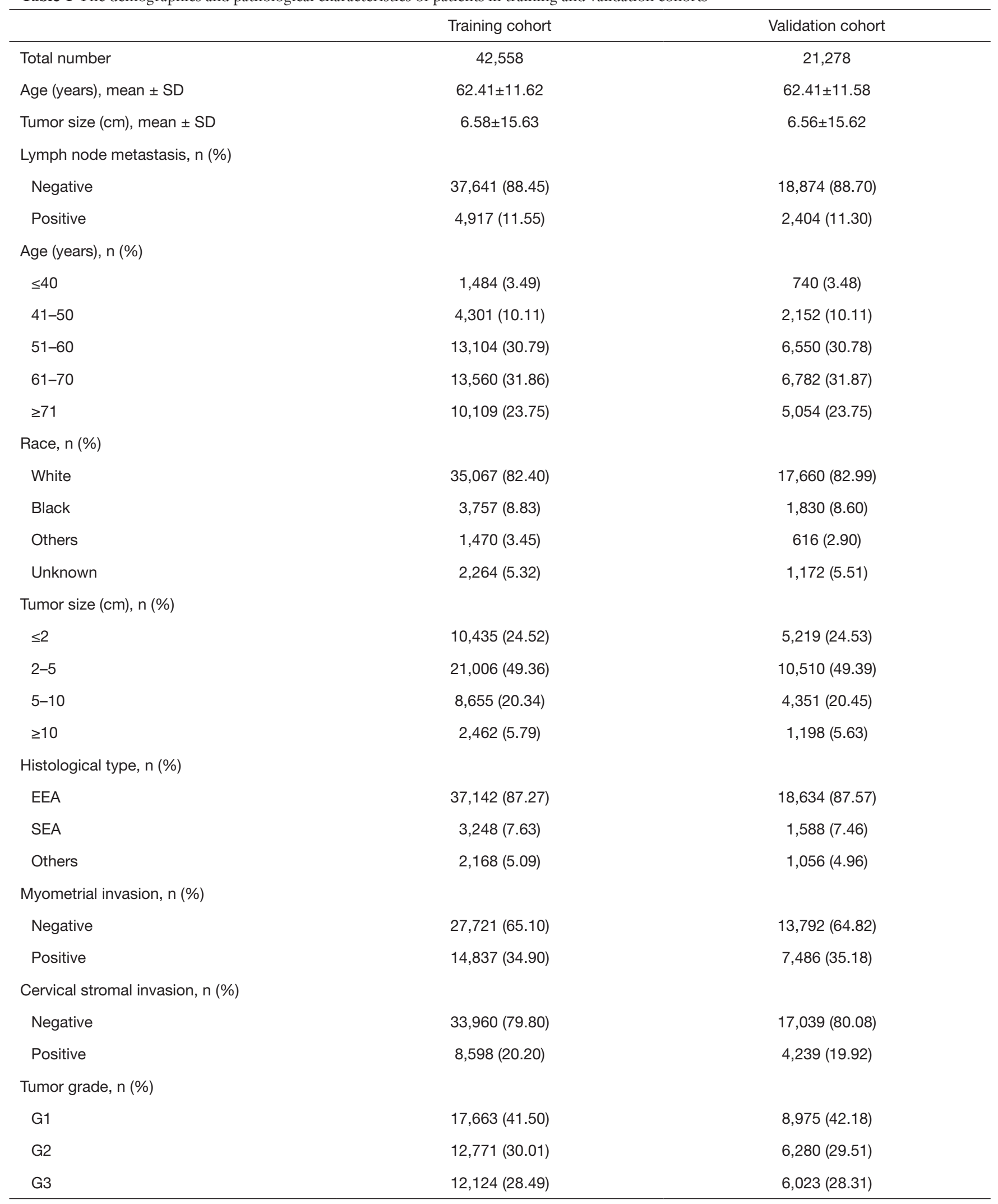


Table 2 Univariate and multivariate analysis of lymph node metastasis in the training cohort

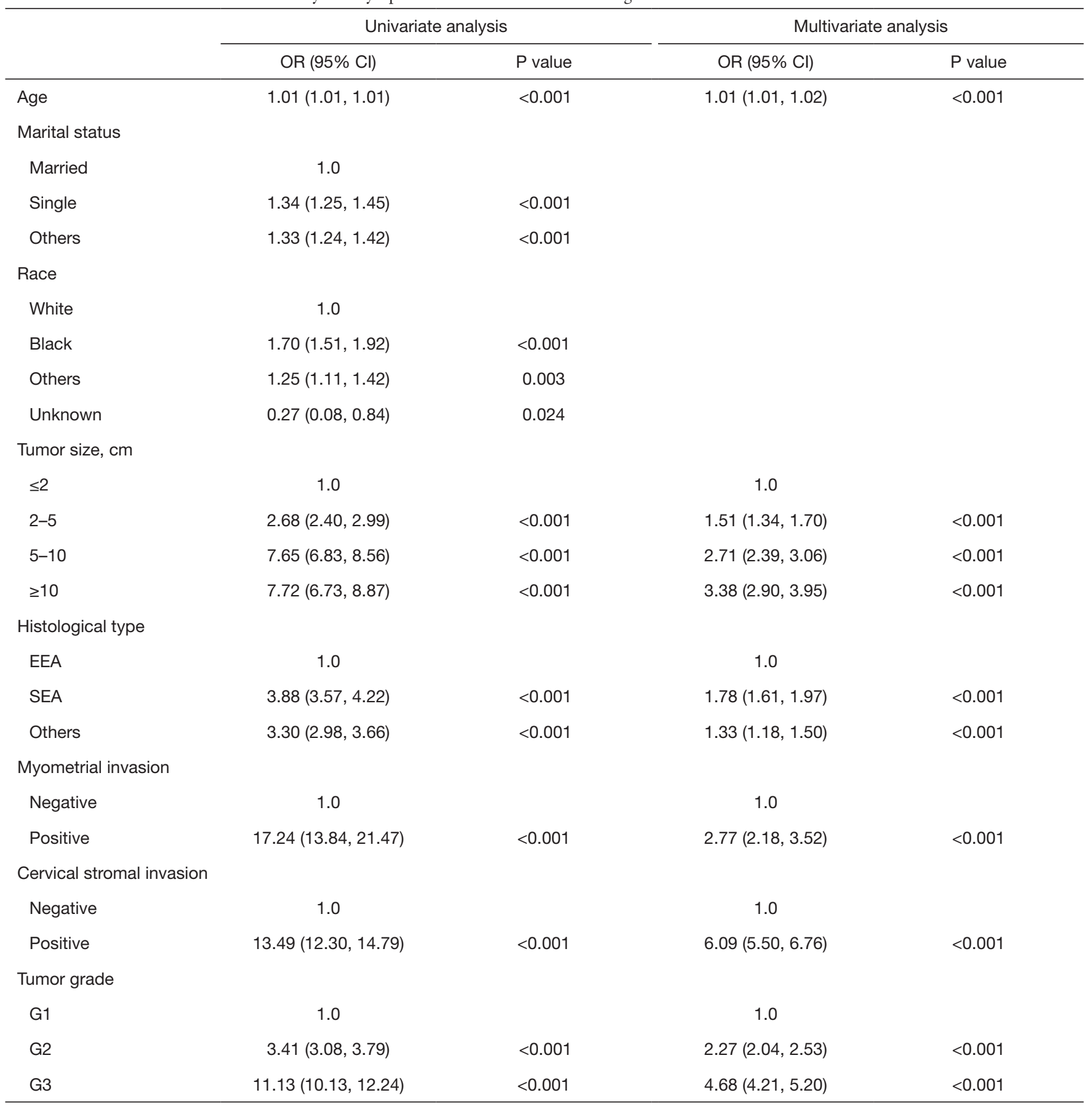

OR, odds ratio; $95 \% \mathrm{Cl}, 95 \%$ confidence interval; EEA, endometrial endometrioid adenocarcinoma; SEA, serous endometrioid adenocarcinoma.

nomogram. Then, an optimal cut-off value of 200 points was selected to maximize sensitivity and specificity of average scores in the ROC curve. Patients from training and validation cohorts were divided into low-risk (score
$<200$ points) and high-risk (score $\geq 200$ points) groups. The performance of the nomogram stratification was compared to the Mayo criteria for predicting LNM. The nomogram showed better discrimination than the Mayo criteria in both 
Table 3 Univariate and multivariate analysis of lymph node metastasis in the validation cohort

\begin{tabular}{|c|c|c|c|c|}
\hline & \multicolumn{2}{|c|}{ Univariate analysis } & \multicolumn{2}{|c|}{ Multivariate analysis } \\
\hline Age & $1.01(1.01,1.02)$ & $<0.001$ & $1.01(1.01,1.02)$ & $<0.001$ \\
\hline \multicolumn{5}{|l|}{ Race } \\
\hline White & 1.0 & & & \\
\hline Others & $1.17(1.04,1.86)$ & 0.005 & & \\
\hline Unknown & $0.15(0.06,0.36)$ & 0.038 & & \\
\hline \multicolumn{5}{|c|}{ Tumor size, $\mathrm{cm}$} \\
\hline$\leq 2$ & 1.0 & & 1.0 & \\
\hline$\geq 10$ & $9.58(7.77,11.80)$ & $<0.001$ & $9.58(7.77,11.80)$ & $<0.001$ \\
\hline \multicolumn{5}{|c|}{ Histological type } \\
\hline EEA & 1.0 & & 1.0 & \\
\hline SEA & $4.09(3.63,4.61)$ & $<0.001$ & $4.09(3.63,4.61)$ & $<0.001$ \\
\hline Others & $2.88(2.47,3.35)$ & $<0.001$ & $2.88(2.47,3.35)$ & $<0.001$ \\
\hline \multicolumn{5}{|c|}{ Myometrial invasion } \\
\hline Negative & 1.0 & & 1.0 & \\
\hline Positive & $19.98(14.22,28.08)$ & $<0.001$ & $19.98(14.22,28.08)$ & $<0.001$ \\
\hline \multicolumn{5}{|c|}{ Tumor grade } \\
\hline G2 & $2.99(2.59,3.45)$ & $<0.001$ & $2.99(2.59,3.45)$ & $<0.001$ \\
\hline G3 & $9.96(8.75,11.34)$ & $<0.001$ & $9.96(8.75,11.34)$ & $<0.001$ \\
\hline
\end{tabular}

OR, odds ratio; $95 \% \mathrm{Cl}$, 95\% confidence interval; EEA, endometrial endometrioid adenocarcinoma; SEA, serous endometrioid adenocarcinoma.

the training (nomogram: AUC $=0.754,95 \%$ CI: $0.747-$ 0.761; Mayo: $\mathrm{AUC}=0.716$, 95\% CI: 0.709-0.723; $\mathrm{P}<0.01$; Figure $5 A$ ) and the validation groups (nomogram: AUC $=0.751,95 \%$ CI: 0.741-0.761; Mayo: AUC $=0.714,95 \%$ CI: $0.706-0.727 ; \mathrm{P}<0.01 ;$ Figure $5 B)$. In the training group, the LNM rates were $4.80 \%$ and $34.0 \%$ in low-risk and high risk groups, respectively, according to the nomogram, and $5.7 \%$ and $26.4 \%$, respectively, according to the Mayo criteria (Table 4). In the validation cohort, the predicted rates of
LNM were $4.8 \%$ and $33.7 \%$ in the low-risk and high-risk groups, respectively, according to the nomogram, and $5.6 \%$ and $25.9 \%$, respectively, according to the Mayo criteria (Table 5).

\section{Decision curve analysis}

The decision curve analysis results for the nomogram and Mayo models are shown in Figure $6 A$ (training cohort) and Figure $6 B$ (validation cohort). For predicted probability 


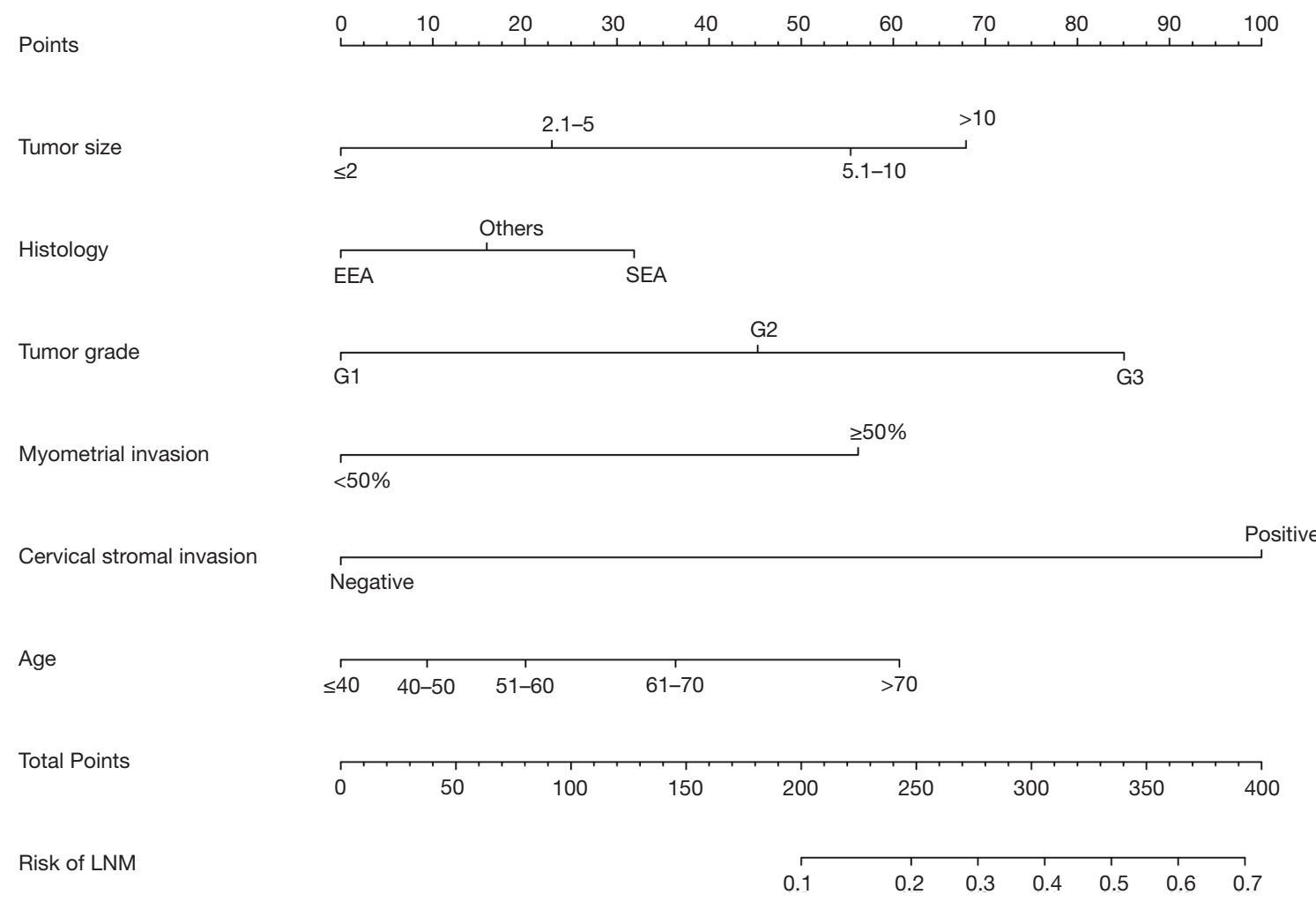

Figure 2 Nomogram to predict lymph node metastasis for EC patients. EC, endometrial cancer.
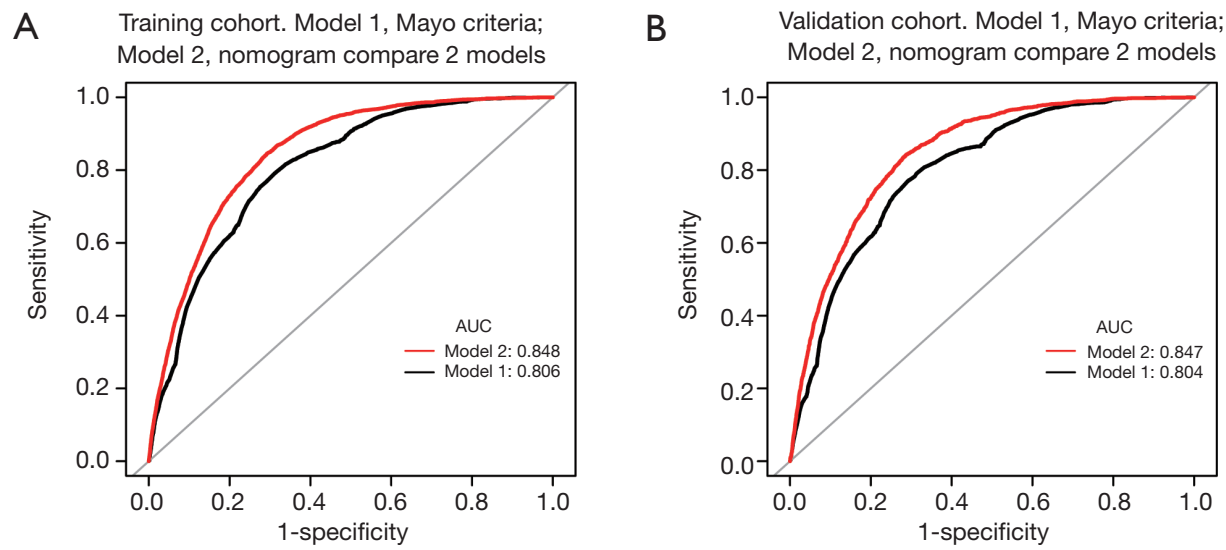

Figure 3 Area under the receiver operating characteristic curves (AUCs) of nomogram and Mayo criteria for the prediction of lymph node metastasis in EC in (A) training cohort and (B) validation cohort. EC, endometrial cancer.

thresholds between $0 \%$ and nearly $60 \%$, the nomogram showed a positive net benefit for both cohorts.

\section{Discussion}

$\mathrm{EC}$ is one of the most common types of gynecologic malignancy. It is estimated that 63.4 out of every 100,000 people in China in 2015 were diagnosed as EC (14). One of the most important prognostic factors for $\mathrm{EC}$ is the presence of LMN. Nevertheless, there is an ongoing debate concerning practicality of lymphadenectomy. Some studies have shown that traditional lymphadenectomy may not 

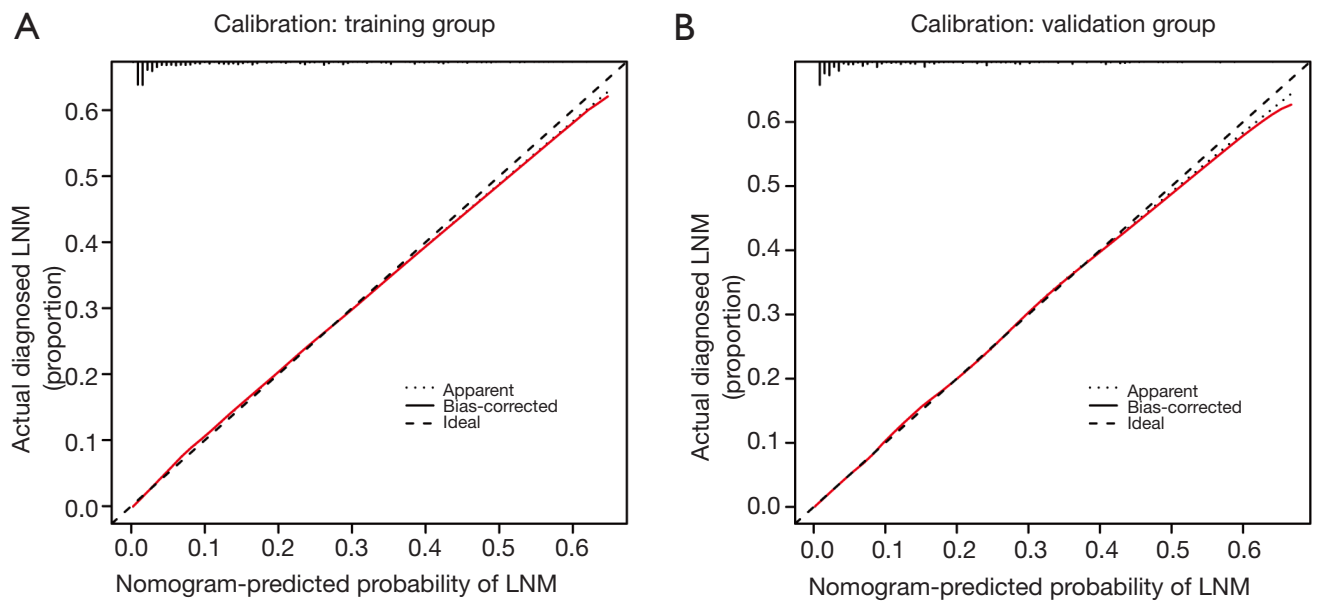

Figure 4 Calibration plots of the nomogram to predict lymph node metastasis in (A) training cohort and (B) validation cohort. In case of perfect calibration, all groups of predicted probabilities fit close to the blue diagnosed line, corresponding to an intercept of 0 and slop of 1 for the calibration plot.
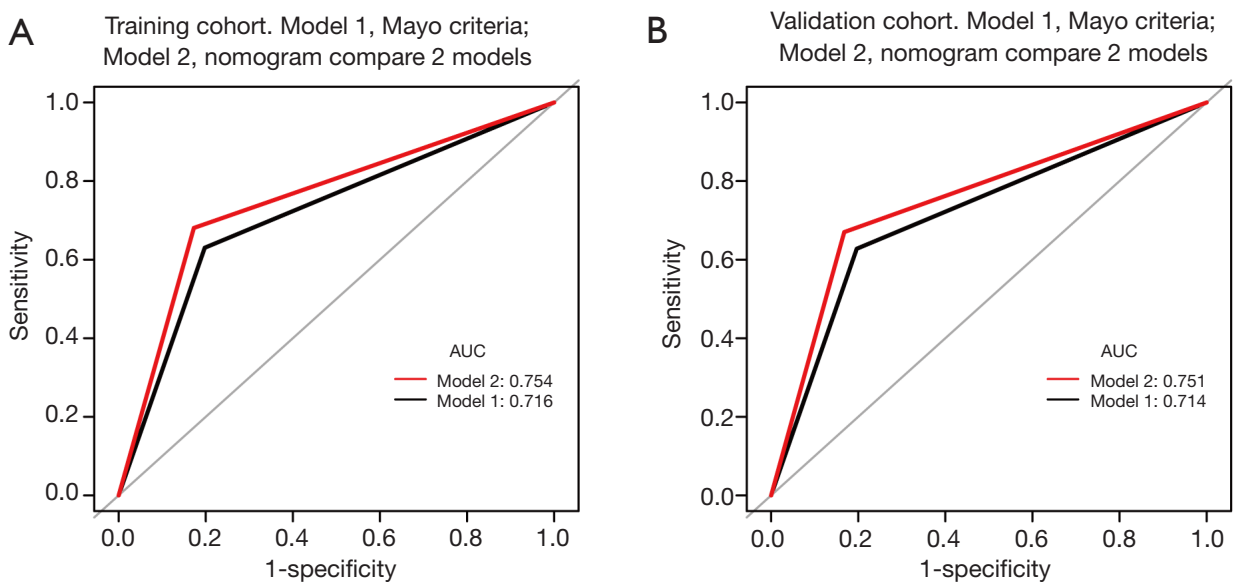

Figure 5 Receiver operating characteristic curve of classification based on nomogram score and Mayo criteria in (A) training cohort and (B) validation cohort.

improve DFS or overall survival, while other studies have suggested the opposite (15-17). Therefore, it is crucial to distinguish patients with low risk of LNM from those with high risk. Currently, most of the studies have followed the Mayo criteria for predicting LNM risk and compared it with different models. According to one study based on the Mayo criteria (18), $78.9 \%$ of the studied patients were at high-risk for nodal metastasis, but the actual $\mathrm{LMN}$ rate was only $6.4 \%$. Thus, almost $70 \%$ patients without $\mathrm{LMN}$ were over-treated. Multiple retrospective studies have shown that a low-risk subset of EC patients have a low overall risk of lymph node involvement (19-21). Mariani et al. performed a large retrospective study of EC patients, which determined that patients with myometrial invasion $<50 \%$, tumor grade 1 or 2 , and tumor size $<2 \mathrm{~cm}$ were at a low risk for lymph node involvement (9). Vargas et al. analyzed 19,329 patients with EC and the results suggested that EC patients for which the Mayo criteria predicted a low risk of LMN, did have a low incidence of metastasis (18). However, the scale of the patients in these studies was relatively small. Therefore, our goal was to conduct a large-cohort study to identify risk factors for LNM and develop accurate risk stratification models to separate patients with EC into lowrisk and high-risk categories. The benefit of this model 
Table 4 Performance or the nomogram and Mayo criteria scoring system in predicting lymph node metastasis in the training cohort

\begin{tabular}{lccc}
\hline Performance parameter & Mayo & Nomogram & P (compare) \\
\hline ROC area (AUC) & 0.7163 & 0.7540 & 0.001 \\
$95 \%$ Cl low & 0.7092 & 0.7472 & 0.7608 \\
$95 \%$ Cl high & 0.7233 & 0.8104 \\
Accuracy & 0.7824 & 0.8273 \\
Specificity & 0.8023 & 0.6807 \\
Sensitivity & 0.6303 & 3.9419 \\
Positive-LR & 3.1882 & 0.3859 \\
Negative-LR & 0.4608 & 0.3399 \\
Positive-PV & 0.2640 & 0.9520 \\
Negative-PV & 0.9432 & PV predictive value. \\
\hline AUC, area under &
\end{tabular}

AUC, area under the receiver operating characteristic curve; LR, likelihood ratio; PV, predictive value.

Table 5 Performance or the nomogram and Mayo criteria scoring system in predicting lymph node metastasis in the validation cohort

\begin{tabular}{|c|c|c|c|}
\hline Performance parameter & Mayo & Nomogram & $\mathrm{P}$ (compare) \\
\hline $95 \% \mathrm{Cl}$ low & 0.7055 & 0.7413 & \\
\hline 95\% Cl high & 0.7256 & 0.7609 & \\
\hline Accuracy & 0.7835 & 0.8138 & \\
\hline Sensitivity & 0.6277 & 0.6701 & \\
\hline Positive-LR & 3.1925 & 3.9912 & \\
\hline Negative-LR & 0.4634 & 0.3964 & \\
\hline Positive-PV & 0.2591 & 0.3370 & \\
\hline
\end{tabular}

AUC, area under the receiver operating characteristic curve; LR, likelihood ratio; PV, predictive value.

will be preventing patients from unnecessary lymph node dissection and the associated surgical morbidity.

Nomograms and clinicopathologic variables have been used by some researchers to estimate risk of metastasis. In the current study, we adopted a large-scale populationbased SEER database to develop and validate a convenient nomogram for doctors to make individualized predictions of LNM in patients with EC. Data were collected for a total of $63,836 \mathrm{EC}$ patients and randomly divided into two groups. Several predictive factors were identified for the SEER patients in this study, including age at diagnosis, race, tumor size, histological type, myometrial invasion, cervical stromal invasion, and tumor grade. Further multivariate logistic regression analysis revealed six factors that were predictive of LNM, including age at diagnosis, tumor size, histological type, myometrial invasion, cervical stromal invasion, and tumor grade. These six factors were incorporated in the nomogram design. The results showed that the nomogram provided good discrimination for both the training (AUC $=0.848 ; 95 \% \mathrm{CI}: 0.843-0.853)$ and validation groups (AUC $=0.847 ; 95 \%$ CI: $0.840-0.857$ ). The nomogram also performed significantly better than the Mayo criteria for predicting LNM in both the training (AUC $=0.806$; 95\% CI: $0.801-0.812, \mathrm{P}<0.001$ ) and validation groups (AUC 
A

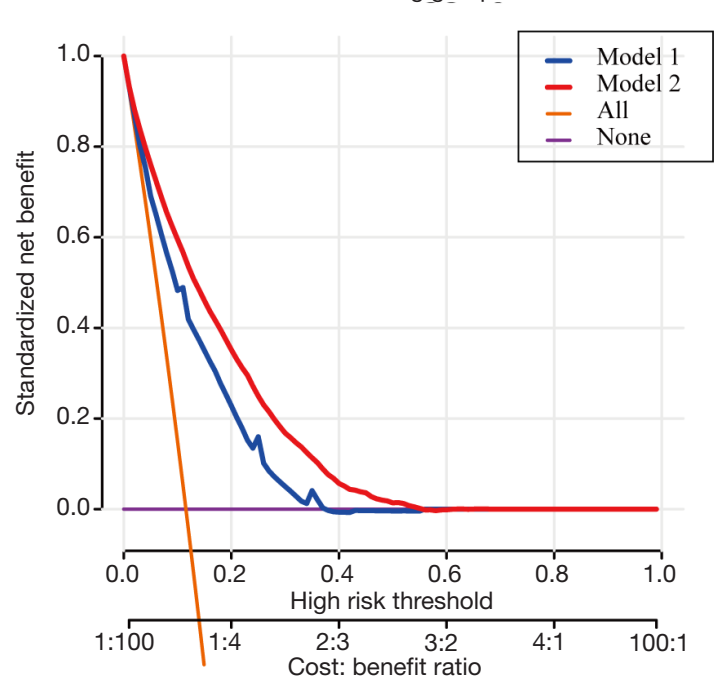

B Net benefit: Validation group

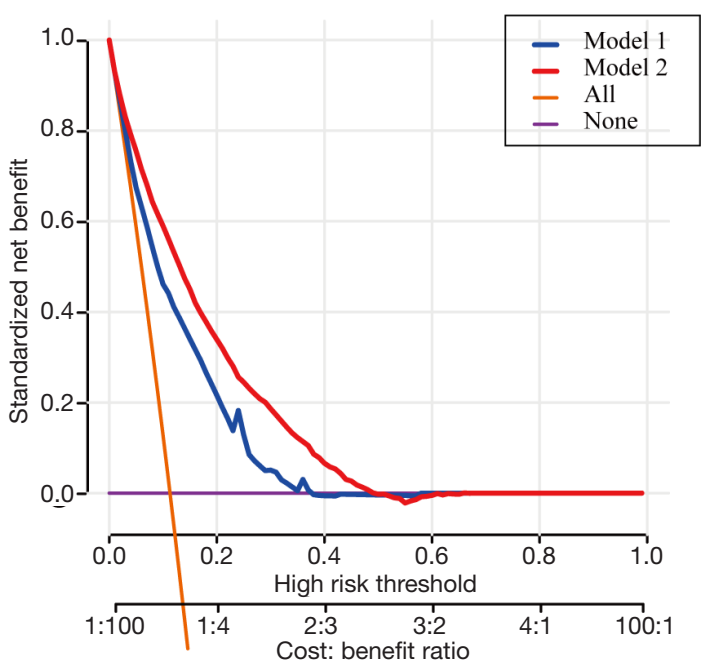

Figure 6 Decision curve analysis of nomogram we developed and Mayo criteria for predicting lymph node metastasis in training cohort and validation cohort. Solid blue line = net benefit when all EC patients are considered as not having the outcome (negative lymph node metastasis); red dashed line = net benefit when all EC patients are considered as having the outcome (positive lymph node metastasis). EC, endometrial cancer.

$=0.804 ; 95 \%$ CI: $0.796-0.813, \mathrm{P}<0.001)$. Furthermore, calibration results indicated that the predictions made using the nomogram fit well with observations for both groups. We used 200 points as the cut-off value based on Youden's index (22) and divided the patients from both cohorts into low-risk (score <200 points) and high-risk groups (score $\geq 200$ points). The performance comparison of our nomogram and Mayo criteria for predicting LNM was verified. The nomogram showed a better discrimination than the Mayo criteria in both training (with AUC of 0.754, 95\% CI: $0.747-0.761$ vs. $0.716,95 \%$ CI: $0.709-0.723$; $\mathrm{P}<0.001$ ) and validation cohorts (with AUC of $0.751,95 \%$ CI: $0.741-0.761$, vs. 0.714 , 95\% CI: $0.706-0.727$; $\mathrm{P}<0.001$ ) for the AUC values were greater for the nomogram than for the Mayo criteria. Decision curve analysis showed that the use of our nomogram had a positive benefit compared to the Mayo criteria. The lymph node metastasis rates were $4.80 \%$ and $34.0 \%$ in low-risk and high-risk groups, respectively, according to the nomogram, while the rates were $5.7 \%$ and $26.4 \%$, respectively, according to the Mayo criteria.

Several previous studies had reported different riskscoring nomograms for predicting LNM. Sofiane constructed a predictive model to identify high-risk LNM patients in early-stage type 1 EC (23). Korean Gynecologic Oncology Group (KGOG) developed a preoperative assessment of LNM in EC that included features of MRI results and serum CA125 levels (24). Lymph-vascular space invasion (LVSI) also had been shown to have a great impact on LNM in some studies $(25,26)$. However, these studies had smaller populations, and thus, their predictive values were lower than the nomogram presented here.

Some researches concentrated on comparing different models. Boyraz et al. compared the Mayo criteria and Milwaukee risk stratification models and found that the Mayo model was more accurate for predicting LNM than the Milwaukee model (27). Tuomi et al. compared the performance characteristics of three risk-stratification models, (Mayo, Helsinki and Milwaukee models) and found that these models had similar accuracies for predicting lymphatic dissemination in EC patients (28).

The nomogram may have performed better than the Mayo model for predicting LNM due to the differences in predictive value assigned to two specific factors in the models: age at diagnosis and cervical stromal invasion (the other four factors performed the same in both models). Age at diagnosis had only a small impact on LNM according to the nomogram algorithm, however, cervical stromal invasion was shown to be a strong predictor of LNM. A previous study from our institute also revealed that cervical stromal invasion is an independent risk factor for LNM (in 
addition to LVSI, tumor grade, and myometrial invasion) in EC patients and may have an important role in predicting LNM (29). Another study also suggested that cervical stromal invasion was useful in estimating LNM risk for EC patients and directing therapeutic strategies (30). This may be because the degree of metastasis and invasion in a particular EC patient was a good predictive indicator of LNM. The stronger the invasive ability of cancer cells, the greater the likelihood of LNM. What's more, according to the 2014 FIGO staging system, cervical stromal invasion causes the cancer to be classified as stage II and the next step in the progression is local and/or regional spread of the cancer (31).

There were several limitations in the present study. First, the study was conducted retrospectively and some selection bias may have occurred. The second limitation was that several critical predictive factors, such as LVSI and menopausal status were unavailable in the SEER database. Third, prospective datasets need to be used to externally validate the nomogram developed from this study. Addressing these areas should be the focus of future research.

\section{Conclusions}

A nomogram based on a large-scale of EC patients for predicting LNM was constructed and validated. Our nomogram showed higher accuracy and net benefit than Mayo criteria for predicting LNM in EC patients.

\section{Acknowledgments}

We especially appreciate Jiayang Jin, PhD of Peking University People's Hospital Rheumatology department for study design consultations and editing the manuscript.

Funding: This study was funded by the grants from the National Natural Science Foundation of China (Grant No. 81874108 and 81802607) and National Key Technology R\&D Program of China (No. 2019YFC1005200 and 2019YFC1005201).

\section{Footnote}

Reporting Checklist: The authors have completed the TRIPOD reporting checklist. Available at http://dx.doi. org/10.21037/atm-20-5034

Conflicts of Interest: All authors have completed the ICMJE uniform disclosure form (available at http://dx.doi. org/10.21037/atm-20-5034). The authors have no conflicts of interest to declare.

Ethical Statement: The authors are accountable for all aspects of the work in ensuring that questions related to the accuracy or integrity of any part of the work are appropriately investigated and resolved. The study was conducted in accordance with the Declaration of Helsinki (as revised in 2013).

Open Access Statement: This is an Open Access article distributed in accordance with the Creative Commons Attribution-NonCommercial-NoDerivs 4.0 International License (CC BY-NC-ND 4.0), which permits the noncommercial replication and distribution of the article with the strict proviso that no changes or edits are made and the original work is properly cited (including links to both the formal publication through the relevant DOI and the license). See: https://creativecommons.org/licenses/by-nc-nd/4.0/.

\section{References}

1. Siegel RL, Miller KD, Jemal A. Cancer statistics, 2018. CA Cancer J Clin 2018;68:7-30.

2. Chan JK, Kapp DS. Role of complete lymphadenectomy in endometrioid uterine cancer. Lancet Oncol 2007;8:831-41.

3. Pecorelli S. Revised FIGO staging for carcinoma of the vulva, cervix, and endometrium. Int J Gynaecol Obstet 2009;105:103-4.

4. Morrow CP, Bundy BN, Homesley HD, et al. Doxorubicin as an adjuvant following surgery and radiation therapy in patients with high-risk endometrial carcinoma, stage I and occult stage II: a Gynecologic Oncology Group Study. Gynecol Oncol 1990;36:166-71.

5. Todo Y, Takeshita S, Okamoto K, et al. Implications of para-aortic lymph node metastasis in patients with endometrial cancer without pelvic lymph node metastasis. J Gynecol Oncol 2017;28:e59.

6. Iasonos A, Schrag D, Raj GV, et al. How to build and interpret a nomogram for cancer prognosis. J Clin Oncol 2008;26:1364-70.

7. Balachandran VP, Gonen M, Smith JJ, et al. Nomograms in oncology: more than meets the eye. Lancet Oncol 2015;16:e173-80.

8. Florkowski CM. Sensitivity, specificity, receiver-operating characteristic (ROC) curves and likelihood ratios: 
communicating the performance of diagnostic tests. Clin Biochem Rev 2008;29 Suppl 1:S83-7.

9. Mariani A, Webb MJ, Keeney GL, et al. Low-risk corpus cancer: is lymphadenectomy or radiotherapy necessary? Am J Obstet Gynecol 2000;182:1506-19.

10. Dowdy SC, Borah BJ, Bakkum-Gamez JN, et al. Prospective assessment of survival, morbidity, and cost associated with lymphadenectomy in low-risk endometrial cancer. Gynecol Oncol 2012;127:5-10.

11. Kilts TP, Glaser GE, Langstraat CL, et al. Comparing risk stratification criteria for predicting lymphatic dissemination in endometrial cancer. Gynecol Oncol 2019;155:21-6.

12. Yang B, Shan B, Xue X, et al. Predicting Lymph Node Metastasis in Endometrial Cancer Using Serum CA125 Combined with Immunohistochemical Markers PR and Ki67, and a Comparison with Other Prediction Models. PLoS One 2016;11:e0155145.

13. Steyerberg EW, Eijkemans MJ, Harrell FE, Jr., et al. Prognostic modelling with logistic regression analysis: a comparison of selection and estimation methods in small data sets. Stat Med 2000;19:1059-79.

14. Chen $W$, Zheng R, Baade PD, et al. Cancer statistics in China, 2015. CA Cancer J Clin 2016;66:115-32.

15. ASTEC study group, Kitchener H, Swart AM, et al. Efficacy of systematic pelvic lymphadenectomy in endometrial cancer (MRC ASTEC trial): a randomised study. Lancet 2009;373:125-36.

16. Coronado PJ, Rychlik A, Martinez-Maestre MA, et al. Role of lymphadenectomy in intermediate-risk endometrial cancer: a matched-pair study. J Gynecol Oncol 2018;29:e1.

17. Venigalla S, Chowdhry AK, Shalowitz DI. Survival implications of staging lymphadenectomy for non-endometrioid endometrial cancers. Gynecol Oncol 2018;149:531-8.

18. Vargas R, Rauh-Hain JA, Clemmer J, et al. Tumor size, depth of invasion, and histologic grade as prognostic factors of lymph node involvement in endometrial cancer: a SEER analysis. Gynecol Oncol 2014;133:216-20.

19. Convery PA, Cantrell LA, Di Santo N, et al. Retrospective review of an intraoperative algorithm to predict lymph node metastasis in low-grade endometrial adenocarcinoma. Gynecol Oncol 2011;123:65-70.

20. Milam MR, Java J, Walker JL, et al. Nodal metastasis risk in endometrioid endometrial cancer. Obstet Gynecol 2012;119:286-92.

21. Shah C, Johnson EB, Everett E, et al. Does size matter? Tumor size and morphology as predictors of nodal status and recurrence in endometrial cancer. Gynecol Oncol 2005;99:564-70.

22. Youden WJ. Index for rating diagnostic tests. Cancer 1950;3:32-5.

23. Bendifallah S, Canlorbe G, Laas E, et al. A Predictive Model Using Histopathologic Characteristics of EarlyStage Type 1 Endometrial Cancer to Identify Patients at High Risk for Lymph Node Metastasis. Ann Surg Oncol 2015;22:4224-32.

24. Kang S, Nam JH, Bae DS, et al. Preoperative assessment of lymph node metastasis in endometrial cancer: A Korean Gynecologic Oncology Group study. Cancer 2017;123:263-72.

25. Koskas M, Bassot K, Graesslin O, et al. Impact of lymphovascular space invasion on a nomogram for predicting lymph node metastasis in endometrial cancer. Gynecol Oncol 2013;129:292-7.

26. AlHilli MM, Podratz KC, Dowdy SC, et al. Risk-scoring system for the individualized prediction of lymphatic dissemination in patients with endometrioid endometrial cancer. Gynecol Oncol 2013;131:103-8.

27. Boyraz G, Atalay FO, Salman MC, et al. Comparison of Mayo and Milwaukee Risk Stratification Models for Predicting Lymph Node Metastasis in Endometrial Cancer. Int J Gynecol Cancer 2018;28:869-74.

28. Tuomi T, Pasanen A, Leminen A, et al. Prediction of lymphatic dissemination in endometrioid endometrial cancer: Comparison of three risk-stratification models in a single-institution cohort. Gynecol Oncol 2017;144:510-4.

29. Dong Y, Cheng Y, Tian W, et al. An Externally Validated Nomogram for Predicting Lymph Node Metastasis of Presumed Stage I and II Endometrial Cancer. Front Oncol 2019;9:1218.

30. Pollom EL, Conklin CM, von Eyben R, et al. Nomogram to Predict Risk of Lymph Node Metastases in Patients With Endometrioid Endometrial Cancer. Int J Gynecol Pathol 2016;35:395-401.

31. Oncology FCoG. FIGO staging for carcinoma of the vulva, cervix, and corpus uteri. Int J Gynaecol Obstet 2014;125:97-8.

Cite this article as: Li X, Cheng Y, Dong Y, Zhou J, Wang Z, Li X, Wang J. Development and validation of predictive model for lymph node metastasis in endometrial cancer: a SEER analysis. Ann Transl Med 2021;9(7):538. doi: 10.21037/atm-205034 